\title{
Neutrino luminosities and heat capacities of neutron stars in analytic form
}

\author{
D. D. Ofengeim, ${ }^{1}$ M. Fortin, ${ }^{2}$ P. Haensel, ${ }^{2}$ D. G. Yakovlev, ${ }^{1}$ and J. L. Zdunik ${ }^{2}$ \\ ${ }^{1}$ Ioffe Institute, 26 Politekhnicheskaya, St. Petersburg 194021, Russia \\ ${ }^{2}$ N. Copernicus Astronomical Center, Bartycka 18, 00-716 Warsaw, Poland
}

(Dated: August 29, 2017)

\begin{abstract}
We derive analytic approximations for the neutrino luminosities and the heat capacities of neutron stars with isothernal nucleon cores as functions of the mass and radius of stars. The neutrino luminosities are approximated for the three basic neutrino emission mechanisms, and the heat capacities for the five basic combinations of the partial heat capacities. The approximations are valid for for a wide class of equations of state of dense nucleonmatter. The results significantly simplify the theoretical interpretation of observations of cooling neutron stars as well as of quasistationary thermal states of neutron stars in X-ray transients. For illustration, we present an analysis of the neutrino cooling functions of nine isolated neutron stars taking into account the effects of their magnetic fields and of the presence of light elements in their heat blanketing envelopes. These results allow one to investigate the superfluid properties of neutron star cores.
\end{abstract}

\section{INTRODUCTION}

Modeling the thermal evolution of isolated neutron stars as well as neutron stars in X-ray transients (XRTs) and comparing the results with observations is known to give a viable method to probe superdense matter in neutron star interiors (e.g., Refs. [1 [5] and references therein).

The cooling of isolated neutron stars proceeds through the neutrino emission from the entire stellar volume and through the heat conduction to the surface and subsequent thermal surface emission of photons. Young and middle-aged isolated neutron stars (of age $t \lesssim 10^{5}$ yr) cool mostly via neutrino emission from their internal layers. Initially, at $t \sim 10-100$ yr after their birth, neutron stars are nonisothermal inside; their cores stay colder than the crust because of the stronger neutrino emission from the cores and the lower thermal conduction in the crust (e.g., Ref. [6] and references therein). However, when the initial period of internal thermal relaxation is over, the interior of the star becomes nearly isothermal. It has the same internal temperature $\widetilde{T}$ which gradually decreases with time as the star cools. With account for the effects of General Relativity, $\widetilde{T}$ should be the temperature redshifted for a distant observer (not the local temperature of the matter, see below). A substantial temperature gradient remains only in the outer heat blanketing envelope of the star (not deeper than a few hundred meters under the surface [7]). The cooling of isolated neutron stars with an isothermal interior at the neutrino cooling stage is governed (e.g., Ref. [3]) by the ratio $L_{\nu}^{\infty}(\widetilde{T}) / C(\widetilde{T})$, where $L_{\nu}^{\infty}(\widetilde{T})$ is the neutrino luminosity of the star (the superscript $\infty$ means that it is redshifted for a distant observer) and $C(\widetilde{T})$ is the heat capacity. Both, $L_{\nu}^{\infty}(\widetilde{T})$ and $C(\widetilde{T})$, are mainly determined by the neutron star core and can be calculated as

$$
L_{\nu}^{\infty}(\widetilde{T})=\int_{0}^{R_{\text {core }}} Q(\rho, T) \frac{\exp (2 \Phi) 4 \pi r^{2} \mathrm{~d} r}{\sqrt{1-2 G m /\left(r c^{2}\right)}}
$$

$$
C_{\text {core }}(\widetilde{T})=\int_{0}^{R_{\text {core }}} c_{\text {core }}(\rho, T) \frac{4 \pi r^{2} \mathrm{~d} r}{\sqrt{1-2 G m /\left(r c^{2}\right)}} .
$$

Here, $Q(\rho, T)$ and $c_{\text {core }}(T, \rho)$ are the neutrino emissivity $\left[\mathrm{erg} \mathrm{cm}^{-3} \mathrm{~s}^{-1}\right]$ and the specific heat capacity [erg $\mathrm{cm}^{-3} \mathrm{~K}^{-1}$ ] in the core, respectively; $\rho$ is the mass (energy) density, $T=\widetilde{T} \exp (-\Phi)$ is the local temperature in the stellar matter, $R_{\text {core }}$ is the core radius, $m=m(r)$ is the gravitational mass inside a sphere of circumferential radius $r$, and $\Phi=\Phi(r)$ is the metric function determined by the equation (e.g., chapter 6 of Ref. [8])

$$
\frac{\mathrm{d} \Phi}{\mathrm{d} r}=-\frac{1}{P+\rho c^{2}} \frac{\mathrm{d} P}{\mathrm{~d} r},
$$

with $P$ being the pressure.

Isolated neutron stars with $10-100 \mathrm{yr} \lesssim t \lesssim 10^{5} \mathrm{yr}$ have isothermal interiors and cool mainly from inside via neutrino emission (e.g., Refs. [3 $[-5])$. To study their cooling, one needs $L_{\nu}^{\infty}(\widetilde{T})$ and $C_{\text {core }}(\widetilde{T})$. Later, at $t \gtrsim 10^{5} \mathrm{yr}$, the neutrino emission weakens and the stars cool mostly via heat conduction to the surface and the surface thermal emission. In order to investigate their cooling at this stage, one needs $C_{\text {core }}(\widetilde{T})$.

Cooling theory is also used to analyze thermal states of neutron stars in XRTs (e.g., Ref. [3]), which are compact binaries containing a neutron star and a low-mass star. These old systems show active periods (days, weeks, or months) of accretion from the low-mass companion to the neutron star through an accretion disk. The active periods are superimposed by quiescent periods (months or years) when the accretion stops.

During an active period, a huge amount of gravitational energy is released when the matter falls onto the neutron star surface. This makes XRTs bright X-ray sources. In addition, the accreted matter burns in thermonuclear reactions in the surface layers which intensifies the surface X-ray emission. The ashes of the thermonuclear burning are further compressed under the weight of the newly accreted material and undergo nuclear transformations (pycnonuclear reactions, electron captures, 
and neutron emission or absorption) producing the deep heating of the neutron star's crust [9 12] with an energy release of about 1-2 MeV per accreted nucleon. This heat, whose power is proportional to the accretion rate, is mainly conducted into the core, warms it up and is radiated by neutrinos from there. As episodes of heating due to the accretion and subsequent quiescence with neutrino cooling proceed, the neutron star interior reaches a state of thermal quasiequilibrium. Then the source operates in a quasistationary regime and the interior of the star remains isothermal because of the large internal thermal conduction. The internal temperature $\widetilde{T}$ does not show noticeable variations since the star is thermally inertial. The star stays thermally balanced being heated during accretion episodes but cooled during quiescent states. In quiescence, the violent processes of surface energy release stop, and the surface temperature drops, but its decrease is limited because the star is still warm inside. Thus the star can produce an intense and detectable quiescent surface emission [12]. The internal temperature $\widetilde{T}$ of this star is determined by its neutrino luminosity $L_{\nu}^{\infty}(\widetilde{T})$ and by the deep crustal heating power (e.g., Ref. [3]).

If accretion episodes are long or intense, the crustal heating can drive the crust out of the thermal balance with the core. This balance is restored later, during subsequent quiescent states. Observations of the relaxation of such neutron stars combined with observations of an accretion outburst allow one to estimate a lower limit to the heat capacity of the neutron star core [13].

Therefore, the thermal evolution of isolated and accreting neutron stars is largely regulated by $L_{\nu}^{\infty}(\widetilde{T})$ and $C(\widetilde{T})$ which are the quantities we analyze below. While modeling the thermal evolution, it is often time consuming to calculate $L_{\nu}^{\infty}(\widetilde{T})$ and $C(\widetilde{T})$ directly from Eqs. (1) and (2). We will obtain convenient analytic fits which considerably simplify the calculations and interpretation of observational data. Note that some fits to $L_{\nu}^{\infty}(\widetilde{T}) / C(\widetilde{T})$ were obtained earlier in Refs. 14, 15]; they are in good agreement with the present results but less complete. Preliminary results of this investigation have been presented in Ref. [16].

\section{BASIC CASES}

The quantities $L_{\nu}^{\infty}(\widetilde{T})$ and $C(\widetilde{T})$ in question are determined (i) by an equation of state (EOS) of superdense matter for the neutron star core and an appropriate neutron star model (for given mass $M$ and radius $R$ ) and (ii) by the neutrino emissivity $Q(\rho, T)$ and the specific heat capacity $c_{\text {core }}(\rho, T)$ in the core. To be specific, we will restrict ourselves to the case in which the core consists of strongly degenerate neutrons $(n)$, protons $(p)$, electrons $(e)$, and muons $(\mu)$, and consider a number of different EOSs of npe $\mu$ matter. As a rule, neutrons are the most abundant particles (e.g., Ref. [8]). Neutrons and protons constitute a strongly interacting Fermi liquid; in the cores
TABLE I. Three basic neutrino processes

\begin{tabular}{lccc}
\hline \hline Case & $L_{\nu}^{\infty}(\widetilde{T})$ & Neutrino process & Superfluids (SF) \\
\hline $\mathrm{DU}$ & $\widetilde{T}^{6}$ & $n \rightarrow p \ell \widetilde{\nu}_{l}, \quad p \ell \rightarrow n \nu_{l}$ & None \\
$\mathrm{MU}$ & $\widetilde{T}^{8}$ & $n N \rightarrow p N \ell \widetilde{\nu}_{l}, \quad p N \ell \rightarrow n N \nu_{l}$ & None \\
$n n$ & $\widetilde{T}^{8}$ & $n n \rightarrow n n \nu \widetilde{\nu}$ & Strong $p$ SF \\
\hline \hline \multicolumn{5}{c}{$N=n$ or $p ; \ell=e$ or $\mu$} \\
\hline \multicolumn{5}{c}{}
\end{tabular}

of massive neutron stars, these particles become mildly relativistic. As for electrons and muons, they constitute a weakly interacting Fermi gas; the electrons are ultrarelativistic while the muons are typically mildly relativistic.

Even in this simplest case, the problem of calculating $L_{\nu}^{\infty}(\widetilde{T})$ and $C(\widetilde{T})$ is strongly complicated by possible superfluidities of neutrons and protons. The critical temperatures for such superfluidities are difficult to calculate exactly (e.g., Ref. [17] and references therein). Neutron and/or proton superfluids affect $L_{\nu}^{\infty}(\widetilde{T})$ and $C(\widetilde{T})$ because of the onset of energy gaps in the nucleon dispersion relations. Strong superfluidity exponentially suppresses the neutrino emissivities for the neutrino processes involving superfluid particles and the partial heat capacities for superfluid particles (as reviewed in Ref. [6]). In addition, it creates a new specific mechanism of neutrinopair emission due to the Cooper pairing of nucleons (see Refs. [4, 5, 18, 19] and references therein). If the superfluid critical temperatures were known, one could in principle numerically compute $L_{\nu}^{\infty}(\widetilde{T})$ and $C(\widetilde{T})$; this could be a good project for the future.

Here, we will follow the strategy used previously in Refs. [14, 15, 20 22 for analyzing the observations of some selected neutron stars. First, we calculate the partial contributions to $L_{\nu}^{\infty}(\widetilde{T})$ and $C(\widetilde{T})$ for the most important cases neglecting the effects of superfluidity. We will approximate these contributions by analytic expressions and use them as the basis for the neutrino luminosities and heat capacities. The actual $L_{\nu}(\widetilde{T})$ and $C(\widetilde{T})$ can be expressed as sums over the contributions from the various species. However if one of them is strongly superfluid then the contribution is strongly reduced (either completely or by an unknwon reduction factor). By comparing the theory with observations using this procedure one can try to constrain the superfluid properties of neutron stars.

Our three basic cases for $L_{\nu}^{\infty}(\widetilde{T})$ are presented in Table [I. where $N$ denotes a nucleon, and $\ell$ is either an electron or a muon. The first column labels the cases, the second one shows the temperature dependence of the corresponding neutrino luminosities $L_{\nu}^{\infty}(\widetilde{T})$, the third presents the leading neutrino reaction and the last column indicates the superfluid state of the neutron star core at which the given $L_{\nu}^{\infty}(\widetilde{T})$ is the leading one. The first case is the most powerful direct Urca (DU) neutrino cooling process 23] (a sequence of neutron decay 
TABLE II. Five basic heat capacities

\begin{tabular}{lc}
\hline \hline Case & $C_{\text {core }}(\widetilde{T})$ \\
\hline$n$ & $C_{n}$ \\
$p$ & $C_{p}$ \\
$\ell$ & $C_{\ell}=C_{e}+C_{\mu}$ \\
tot & $C_{\text {tot }}=C_{n}+C_{p}+C_{e}+C_{\mu}$ \\
$n \ell$ & $C_{n \ell}=C_{n}+C_{e}+C_{\mu}$ \\
\hline \hline
\end{tabular}

and inverse reactions producing an electron or a muon neutrino pair). The DU process can be open only in the inner cores of massive neutron stars with those EOSs which predict a sufficiently large fraction of protons. The second case corresponds to a less powerful neutrino cooling due to the modified Urca (MU) process (e.g. Ref. [6] and references therein). This process is considered as standard in not very massive nonsuperfluid neutron stars. Finally, the last case $n n$ is for the neutrino-pair bremsstrahlung due to neutron-neutron collisions (reviewed, e.g., in Ref. [6]). In a nonsuperfluid star it is weaker than the MU, but if protons are strongly superfluid, MU and DU processes are greatly suppressed and the neutrino-pair bremsstrahlung becomes the leading neutrino cooling process.

Table II presents the five basic cases of partial (nonsuperfluid) heat capacities $C_{\text {core }}(\widetilde{T})$ in neutron star cores. Case $n$ refers to the heat capacity of neutrons; case $p$ to the heat capacity of protons; case $\ell$ to the sum of heat capacities of electrons and muons, case tot to the sum of the heat capacities of all constituents of the matter, and case $n \ell$ to the heat capacity of neutrons, electrons and muons. Case tot gives the total heat capacity of a nonsuperfluid core. Other cases can be useful in the presence of superfluidity of nucleons. For instance, case $n \ell$ corresponds to a strong superfluidity of protons, while case $\ell$ to a strong superfluidity of neutrons and protons. All basic heat capacities are proportional to $\widetilde{T}$ because of the strong degeneracy of all fermions in the neutron star cores (e.g., Ref. [6]).

\section{A. Neutrino luminosities}

Let us outline the neutrino emissivities $Q(\rho, T)$ for the three basic neutrino emission cases in Table I (e.g., Ref. [6]).

For the MU process,

$$
Q_{\mathrm{MU}}=Q_{\mathrm{MU} 0}\left(\frac{n_{p}}{n_{0}}\right)^{1 / 3} T_{9}^{8} \Omega\left(n_{n}, n_{p}, n_{e}, n_{\mu}\right),
$$

where $n_{\alpha}$ is the number density of particles $\alpha=n, \ldots, \mu$; $n_{0}=0.16 \mathrm{fm}^{-3}$ is the standard number density of nucleons in saturated nuclear matter, $T_{9}$ is the local temperature $T$ the expressed in $10^{9} \mathrm{~K}$ and $\Omega \sim 1$ is a di- mensionless factor to account for the different branches of the process (e.g., Refs. 6, 24 $)$. Here we only need the main dependence $Q_{\mathrm{MU}} \propto n_{p}^{1 / 3}$. The factor $Q_{\mathrm{MU} 0} \approx$ $1.75 \times 10^{21} \mathrm{erg} \mathrm{cm}^{-3} \mathrm{~s}^{-1}$ (as well as similar factors for other processes) is calculated under the assumptions described in Ref. [6], with the effective masses of nucleons $m_{p}^{*}=0.7 m_{p}$ and $m_{n}^{*}=0.7 m_{n}$. The difference between the effective and bare masses of nucleons in neutron star cores is mainly determined by the many-body effects.

In the case of the DU process,

$$
Q_{\mathrm{DU}}=Q_{\mathrm{DU} 0}\left(\frac{n_{e}}{n_{0}}\right)^{1 / 3} T_{9}^{6}\left(\Theta_{n p e}+\Theta_{n p \mu}\right)
$$

where $Q_{\mathrm{DU} 0} \approx 1.96 \times 10^{27} \mathrm{erg} \mathrm{cm}^{-3} \mathrm{~s}^{-1}$. The factors $\Theta_{n p e}$ and $\Theta_{n p \mu}$ are equal to 1 (open the electron and muon processes, respectively) if the Fermi momenta of the reacting particles satisfy the corresponding triangle condition; otherwise, these factors are zero. Because of the triangle conditions, the electron and muon DU processes have thresholds and can operate only in the central regions of massive neutron stars.

In the case $n n$ (of strongly superfluid protons; e.g., Ref. [15]),

$$
Q_{n n}=Q_{n n 0}\left(\frac{n_{n}}{n_{0}}\right)^{1 / 3} T_{9}^{8}
$$

with $Q_{n n 0} \approx 1.77 \times 10^{19} \mathrm{erg} \mathrm{cm}^{-3} \mathrm{~s}^{-1}$.

\section{B. Heat capacities}

Now we outline the specific heat capacities in neutron star cores for the basic cases listed in Table II using the well-known expressions presented, for instance, in Ref. [3].

The total specific heat is

$$
c_{t o t}=c_{n}+c_{p}+c_{e}+c_{\mu} .
$$

For any fermion species $\alpha=n, \ldots \mu$,

$$
c_{\alpha}=\frac{k_{B}^{2}}{3 \hbar^{3}} T m_{\alpha}^{*} p_{F \alpha},
$$

where $k_{B}$ is the Boltzmann constant; $m_{\alpha}^{*}$ and $p_{F \alpha}$ are, respectively, the effective mass and the Fermi momentum of the particles $\alpha$. Note that the main contributions to the heat capacity of nonsuperfluid cores come from the neutrons and protons (e.g., Ref. 25]). Assuming again $m_{n}^{*}=0.7 m_{n}$ and $m_{p}^{*}=0.7 m_{p}$, we obtain

$$
c_{N} \approx c_{0}\left(\frac{n_{N}}{n_{0}}\right)^{1 / 3} T_{9},
$$

with $c_{0}=1.12 \times 10^{20} \mathrm{erg} \mathrm{cm}^{-3} \mathrm{~K}^{-1}$. 


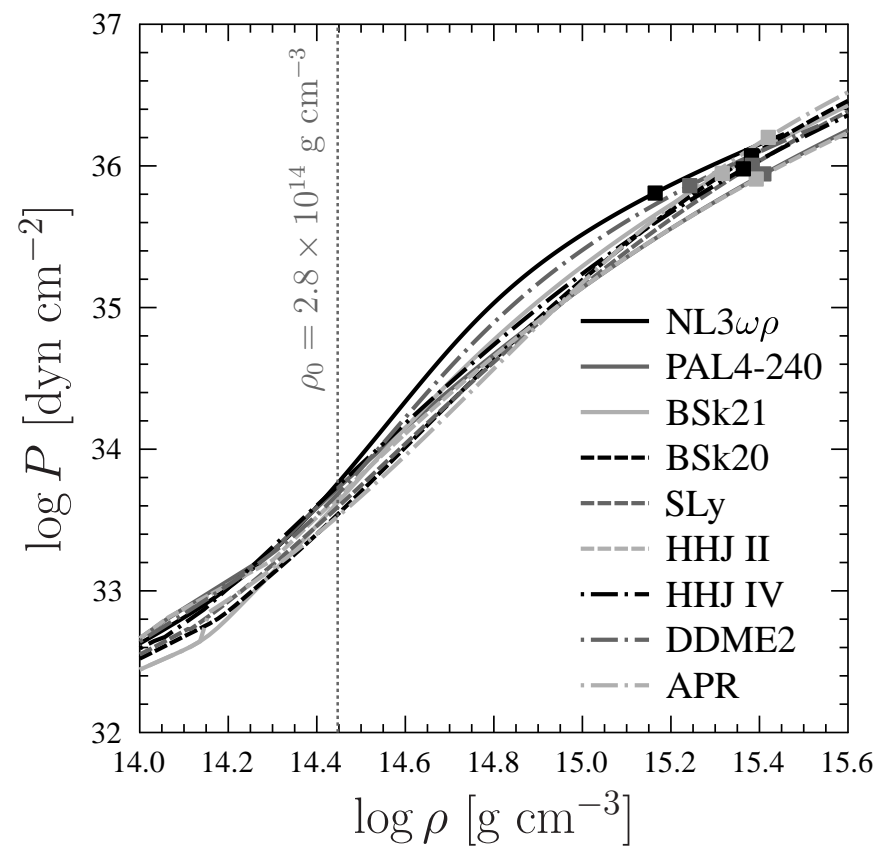

FIG. 1. Plots of the selected EOSs, $P=P(\rho)$, in neutron star cores. Squares mark the maximum central densities of stable neutron stars. See text for details.

The effective mass of ultrarelativistic, nearly ideal electrons $m_{e}^{*}=p_{F e} / c$ is determined by the relativistic effects. Then

$$
c_{e} \approx 0.355 c_{0}\left(\frac{n_{e}}{n_{0}}\right)^{2 / 3} T_{9} .
$$

Since the muons are mildly relativistic, the expression for $c_{\mu}$ is more complicated but the muon contribution in Eq. (7) can be roughly taken into account by an artificial amplification of $c_{e}$.

\section{GRID OF EQUATIONS OF STATE}

In order to calculate $L_{\nu}^{\infty}$ and $C_{\text {core }}$, we have selected nine EOSs of matter in neutron star cores. They are listed in Table $\amalg$ and illustrated in Figs. 2 and 3. The NL3 $\omega \rho$ and DDME2 EOSs were described in Ref. 26] and in references therein. The SLy EOS was calculated in Ref. 27]. The PAL4-240 EOS was constructed using the results of Ref. 28] but with a different compression modulus of symmetric nuclear matter at saturation, $K_{0}=240 \mathrm{MeV}$ (this EOS was also presented in Appendix D of Ref. [8] where it was called the PAPAL EOS). The HHJ II EOS was introduced in Ref. [29] (where it was called the APR II EOS). The BSk20 and BSk21 EOSs have been detailed and parametrized in Ref. [30]. The HHJ IV EOS was built in Ref. [31]. The APR EOS was constructed in Ref. [32]. Let us stress that the selected EOSs are based on essentially different many-body theo-

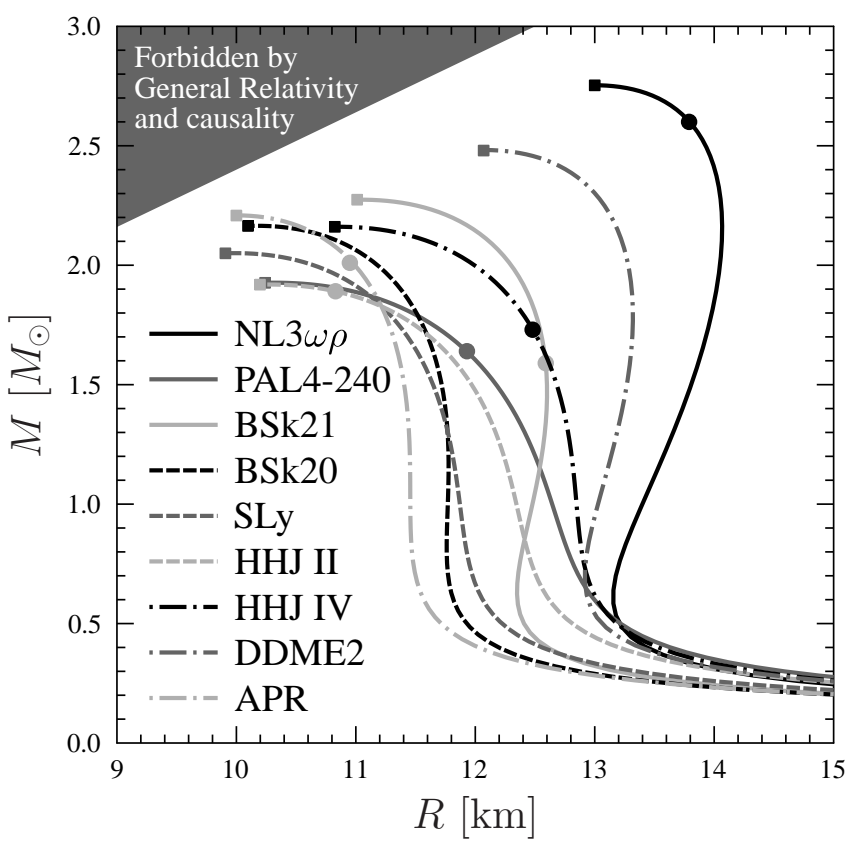

FIG. 2. $M-R$ relations for the EOSs from Fig. 1] Filled squares mark the maximum masses of stable neutron stars; filled circles mark the threshold masses of stars in which the DU process is open (Table III).

TABLE III. The basic parameters of neutron stars for the selected EOSs; $M_{\max }$ and $R_{\min }$ refer to the most massive stable stars; $M_{\mathrm{DU}}$ and $R_{\mathrm{DU}}$ refer to the stars where the DU process becomes allowed; $M_{\odot}$ is the mass of the Sun.

\begin{tabular}{lcccc}
\hline \hline EOS & $M_{\max }, M_{\odot}$ & $R_{\min }, \mathrm{km}$ & $M_{\mathrm{DU}}, M_{\odot}$ & $R_{\mathrm{DU}}, \mathrm{km}$ \\
\hline NL3 $\rho$ & 2.75 & 13.00 & 2.60 & 13.79 \\
PAL4-240 & 1.93 & 10.24 & 1.64 & 11.93 \\
BSk21 & 2.27 & 11.01 & 1.59 & 12.59 \\
BSk20 & 2.16 & 10.10 & - & - \\
SLy & 2.05 & 9.91 & - & - \\
HHJ II & 1.92 & 10.20 & 1.89 & 10.83 \\
HHJ IV & 2.16 & 10.82 & 1.73 & 12.48 \\
DDME2 & 2.48 & 12.07 & - & - \\
APR & 2.21 & 10.00 & 2.01 & 10.95 \\
\hline \hline
\end{tabular}

ries of dense matter. We have selected them to extend a class of basically different EOSs.

To construct neutron star models, one needs also an EOS in the crust. For the SLy, BSk20 and BSk21 models, the EOSs in the crust and the core were calculated in a unified way. The crustal EOSs for the NL3 $\omega \rho$ and DDME2 models were described in Ref. [26]. The APR EOS, originally valid in the core only, has been supplemented with the crustal part of the BSk21 EOS. For the other models, a crust EOS with a smooth composition 


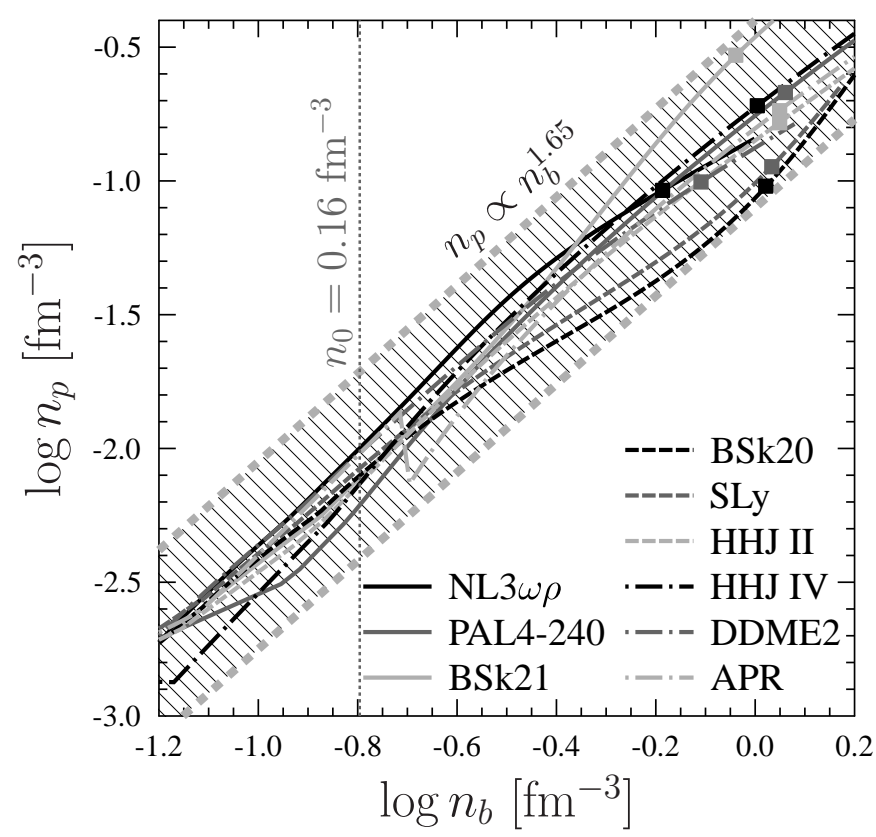

FIG. 3. $n_{p}-n_{b}$ relations for the selected EOSs. Squares mark the maximum $n_{b}$ in stable neutron stars. The thick shaded strip corresponds to the power-law models $n_{p} \propto n_{b}^{1.65}$. See text for details.

[8] has been used.

The selected EOSs $(P=P(\rho))$ in the neutron star cores are plotted in Fig. 1. Near the saturation density $\rho_{0}$ of the standard symmetric nuclear matter, $\rho \sim \rho_{0}=$ $2.8 \times 10^{14} \mathrm{~g} \mathrm{~cm}^{-3}$ (the dotted vertical line), they are not very different. This is because the EOSs are usually constructed in such a way to reproduce the properties of saturated nuclear matter which are well studied in the laboratory.

The most important parameters of neutron stars for the selected EOSs are listed in Table III. The $M(R)$ relations for the neutron star models with these EOSs are plotted in Fig. 2. Since we have chosen EOSs which are sufficiently diverse and have different stiffnesses at $\rho \gtrsim 2 \rho_{0}$, they result in different $M(R)$ relations and cover a large part of the $M-R$ plane. The squares in Fig. 2 correspond to the most massive stable neutron stars. The selected EOSs are consistent with the recent discoveries [33 35] of two massive $\left(M \approx 2 \mathrm{M}_{\odot}\right)$ neutron stars. Circles mark the configurations where the DU process becomes allowed in the neutron star center. Only three EOSs from Table III prohibit the DU process for all stable neutron stars.

Figure 3 illustrates another important property of the selected EOSs: the relation between the proton $n_{p}$ and the total baryon $n_{b}=n_{n}+n_{p}$ number densities. The spread of the curves for the different EOSs is the thinnest at $n_{b} \sim n_{0}$ (the dotted vertical line). It is a consequence of the calibration of the EOSs to the standard nuclear theory (see above). The straight thick shaded strip cor- responds to the relations $n_{p} \propto n_{b}^{1.65}$. In this way we improve the formula $n_{p}\left(n_{b}\right)$ with the $n_{p} \propto n_{b}^{2}$ dependence characteristic of the free Fermi gas model with nonrelativistic nucleons (e.g., Refs. 36, 37]). A smaller powerlaw index effectively accounts for nucleon interactions. According to Fig. 3, this simple approximation is qualitatively accurate; it appears sufficient for our analysis.

Note that we do not intend to accurately fit the EOSs or number densities of the different particles. Our aim is to suggest some simple scaling expressions for these quantities and use them to describe the integral quantities, $C_{\text {core }}$ and $L_{\nu}^{\infty}$. One can treat these scaling expressions as purely phenomenological but we prefer to introduce them on physical grounds. We will see that the integration over the core absorbs the inaccuracy of the scaling expressions and helps to accurately describe $C_{\text {core }}$ and $L_{\nu}^{\infty}$ as functions of $M$ and $R$ for the entire collection of EOSs.

\section{CALCULATION OF THE NEUTRINO LUMINOSITY AND HEAT CAPACITY}

\section{A. Basic remarks}

We have numerically calculated $L_{\nu}^{\infty}(\widetilde{T})$ and $C_{\text {core }}(\widetilde{T})$ from Eqs. (1) and (2) for the three basic models of neutrino emission (Table I) and the five basic models of heat capacity (Table III). We have used a dense grid of neutron star models with different $M$ for all nine EOSs from our collection (Sec. III, Table III). In the calculations, we have used accurate expressions for $Q(\rho, T)$ and $c_{\text {core }}(\rho, T)$ from Ref. 14 which are also employed in our standard cooling code [38] (taking $m_{N}^{*}=0.7 m_{N}$, to be specific).

Our calculation of $L_{\mathrm{DU}}^{\infty}(\widetilde{T})$ deserves a comment. As discussed above, the DU process opens only at high densities in the inner cores of massive stars. To simplify our analysis, we have used $Q=Q_{\mathrm{DU}}$ throughout the entire neutron star core (to avoid complications with the introduction of the DU threshold). This simplification is qualitatively justified because, typically, $Q_{\mathrm{DU}} \sim 10^{6} Q_{\mathrm{MU}}$ (e.g., Ref. [6]), and even a small central kernel with the allowed DU process makes $L_{\mathrm{DU}}^{\infty}(\widetilde{T})$ much larger than $L_{\mathrm{MU}}^{\infty}(\widetilde{T})$. However, it somewhat overestimates $L_{\mathrm{DU}}^{\infty}(\widetilde{T})$ and only gives its firm upper limit. With this simplification, in all our basic cases $L_{\alpha}^{\infty}(\widetilde{T})$ and $C_{\alpha}(\widetilde{T})$ have a predetermined $\widetilde{T}$ dependence (Sec. III), so it is enough to choose one value of $\widetilde{T}$ and calculate $L_{\alpha}^{\infty}(\widetilde{T})$ and $C_{\alpha}(\widetilde{T})$ for the different masses $M$ and EOSs. The selected grid of masses was $M=1.0 M_{\odot}, 1.1 M_{\odot}, \ldots M_{\max }$. As mentioned above, while calculating $L_{\mathrm{DU}}^{\infty}$ we have extended $Q_{\mathrm{DU}}$ over the entire core, but in this case we have not used stellar models with $M<1.5 M_{\odot}$ because $M_{\mathrm{DU}}>1.5 M_{\odot}$ for all our models (Table $\amalg$ ). 


\section{B. Analytic approximations for $L_{\nu}^{\infty}$ and $C_{\text {core }}$}

The exact analytic integration in Eqs. (11) and (2) is not possible. Instead, let us derive some approximate expressions for $L_{\nu}^{\infty}(\widetilde{T})$ and $C_{\text {core }}(\widetilde{T})$ and calibrate them using numerical results.

The first step is to assume that the main contribution to the baryon number density $n_{b}=n_{n}+n_{p}$ is provided by the neutrons. Using a simple model $n_{p} \propto n_{b}^{1.65}$, we get

$$
n_{n} \approx n_{b}, \quad n_{p} \approx n_{e} \approx a n_{0}\left(\frac{n_{b}}{n_{0}}\right)^{1.65} .
$$

Here $a$ is a dimensionless constant which we treat as a value averaged over all selected EOSs. The approximation $n_{e} \approx n_{p}$ can be significantly violated at very high densities at which $n_{\mu} \sim n_{\mathrm{e}}$ (in central regions of massive neutron stars). Their contributions to the integrated neutrino luminosities and heat capacities can be approximately described by an artificial amplification of the electron contributions. For $L_{\mathrm{DU}}^{\infty}(\widetilde{T})$, the contributions of the muon and electron DU processes are just equal.

According to Table $[$ we study the three cases $(\alpha=n n$, MU and DU) of $L_{\alpha}^{\infty}(\widetilde{T})$ in Eq. (1). To find an approximate expression for $L_{n n}^{\infty}(\widetilde{T})$, we take $Q=Q_{n n}$ from Eq. (6). In the second case $(\alpha=\mathrm{MU})$ we employ $Q_{\mathrm{MU}}$ from Eq. (4) with $\Omega=$ const. In the case $\alpha=\mathrm{DU}$ we use Eq. (5) but replace the sum of $\Theta$ functions by a factor of 2 . Since typical densities at which the DU processes operate are so high that the muons appear, this simplification is reasonable.

Then $L_{\nu}^{\infty}(\widetilde{T})$ can be evaluated with the midpoint method, taking the integrand at some fixed value of $r=r^{*}$ between 0 and $R_{\text {core }}$,

$L_{\nu}^{\infty}=Q_{0} R^{3} \widetilde{T}_{9}^{n} \times a^{\prime}\left(\frac{R_{\text {core }}}{R}\right)^{3} \frac{\left(n_{b *} / n_{0}\right)^{k / 3} \exp \left[(2-n) \Phi_{*}\right]}{\sqrt{1-2 G m_{*} /\left(r_{*} c^{2}\right)}}$.

Here $k=1, n=8$ and $Q_{0}=Q_{n n 0}$ for $\alpha=n n ; k=1.65$, $n=8$ and $Q_{0}=Q_{\mathrm{MU} 0}$ for $\alpha=\mathrm{MU} ; k=1.65, n=6$ and $Q_{0}=Q_{\mathrm{DU} 0}$ for $\alpha=\mathrm{DU}$. In Eq. (12) we have introduced a dimensionless constant $a^{\prime}$ to absorb the inaccuracy of $L_{\nu}^{\infty}$ due to our approximations of $n_{p}\left(n_{b}\right)$ and $\Omega$ in the $\mathrm{DU}$ and MU cases; in the $n n$ case, $a^{\prime}=1$. The midpoint values $n_{b *}, \Phi_{*}$ and $m_{*}$ are taken at a spherical shell with $r=r_{*}$.

The five basic cases of heat capacity (Table III) can be presented in a similar way,

$$
C_{\text {core }}=c_{0} R^{3} \widetilde{T}_{9} \times b^{\prime}\left(\frac{R_{\text {core }}}{R}\right)^{3} \frac{\left(n_{b *} / n_{0}\right)^{k / 3} \exp \left(-\Phi_{*}\right)}{\sqrt{1-2 G m_{*} /\left(r_{*} c^{2}\right)}} .
$$

According to Eq. (9), in the case of $C_{n}$ we have $k=1$ and $b^{\prime}=1$. In the case of $C_{p}$ from Eq. (11) we employ $k=1.65$. As for the case of $C_{\ell}$, we assume that the main contribution comes from the electrons, Eq. (10); using
Eq. (11) we set $k=2 \times 1.65=3.30$. The constants $b^{\prime}$ in the two latter cases are thought to absorb the inaccuracies of these analytic approaches. The heat capacities $C_{t o t}$ and $C_{n \ell}$ are thought to be mainly determined by the neutrons. Then we set $k=1$ for both cases and assume that tuning $b^{\prime}$ will make the approximations sufficiently accurate.

The next step is to consider a polytropic EOS model, $P=K \rho^{\gamma}$, which is a primitive but useful approximation. Since all particles in the core are strongly degenerate,

$$
P=c^{2} n_{b}^{2} \frac{\mathrm{d}\left(\rho / n_{b}\right)}{\mathrm{d} n_{b}} .
$$

Then, using the polytropic relation and assuming the boundary condition $\mathrm{d} \rho / \mathrm{d} n_{b}=m_{0}$ at the neutron star surface we get, for catalyzed neutron star matter,

$$
n_{b}=\frac{\rho}{m_{0}}\left(1+\frac{K}{c^{2}} \rho^{\gamma-1}\right)^{-1 /(\gamma-1)} .
$$

Here $m_{0}$ is the mass per baryon in the ${ }^{56} \mathrm{Fe}$ atom. For any neutron star, we can find $\rho_{*}, K$ and $\gamma$ to evaluate $n_{b *}$ needed in Eqs. (12) and (13). Note that $\gamma$ is specific to a given star; it can vary with growing $M$.

Now we are ready to evaluate the relation between $\Phi_{*}$ and $\rho_{*}$. Using Eq. (15) one can solve Eq. (3) and obtain

$$
\Phi=\frac{1}{2} \ln \left(1-x_{g}\right)-\ln \left(\frac{P+\rho c^{2}}{m_{0} c^{2} n_{b}}\right) .
$$

Here we have used the boundary value $\Phi_{s}=\frac{1}{2} \ln (1-$ $\left.x_{g}\right)$ at the stellar surface, with $x_{g}=2 G M /\left(R c^{2}\right)$. The polytropic approach yields

$$
\exp \Phi_{*}=\sqrt{1-x_{g}}\left(1+\frac{K}{c^{2}} \rho_{*}^{\gamma-1}\right)^{-\gamma /(\gamma-1)} .
$$

The quantity $\xi(M, R)=R / R_{\text {core }}$ can be taken from Refs [39, 40] as

$$
\xi(M, R) \equiv \frac{R}{R_{\text {core }}}=1 / x_{g}-\exp \left(-2 \chi_{c c}\right)\left(1 / x_{g}-1\right),
$$

where $\chi_{c c}=\int_{0}^{P_{c c}} \mathrm{~d} P /\left(P+\rho c^{2}\right)$ is an integral over the crust and $P_{c c}$ is the pressure at the core/crust interface. For catalyzed matter, one has $\exp \left(\chi_{c c}\right)=\mu_{c c} / m_{0} c^{2}$, where $\mu$ is the baryon chemical potential. The value $\chi_{c c}$ slightly varies from one EOS to another. Here we adopt $\chi_{\mathrm{cc}}=0.03$ as a unified value for all the EOSs of our study.

To approximate $L_{\nu}^{\infty}(\widetilde{T})$ and $C_{\text {core }}(\widetilde{T})$ we should substitute Eqs. (15), (17) and (18) into Eqs. (12) and (13) and fix $a^{\prime}, b^{\prime}, \rho_{*}, m_{*}, r_{*}, \gamma$ and $K$. Let us introduce $x_{\rho}=M /\left(\rho_{0} R^{3}\right)$ and assume that $\rho_{*} \sim M / R_{\text {core }}^{3}$. Thus 
TABLE IV. Parameters of the approximations (20) and (21) for the three basic models of neutrino luminosity $L_{\nu}^{\infty}$ and the five basic models of heat capacity $C_{\text {core }}$.

\begin{tabular}{ccccccccccccc}
\hline \hline$L_{\nu}^{\infty}$ or $C_{\text {core }}$ & Case & $Q_{0}^{\dagger}$ or $c_{0}^{\ddagger}$ & $n$ & $k$ & $p$ & $a_{1}$ & $a_{2}$ & $a_{3}$ & $a_{4}$ & $a_{5}$ & rms & max error \\
\hline \multirow{6}{*}{$L_{\nu}^{\infty}$} & $n n$ & $1.77 \times 10^{19}$ & 8 & 1 & 6 & 3.54 & 0.0125 & 2.73 & 4.33 & 0.509 & 0.05 & 0.17 \\
& $\mathrm{MU}$ & $1.75 \times 10^{21}$ & 8 & 1.65 & 6 & 2.05 & 0.0125 & 2.58 & 4.40 & 0.480 & 0.15 & 0.42 \\
& $\mathrm{DU}$ & $1.96 \times 10^{27}$ & 6 & 1.65 & 4 & 1.80 & 0.0070 & 2.62 & 4.80 & 0.501 & 0.08 & 0.20 \\
\hline \multirow{6}{*}{$C_{\text {core }}$} & $n$ & $1.12 \times 10^{20}$ & 1 & 1 & 1 & 2.86 & 0.0119 & 2.49 & 3.68 & 0.408 & 0.0084 & 0.025 \\
& $p$ & $1.12 \times 10^{20}$ & 1 & 1.65 & 1 & 0.781 & 0.0069 & 2.70 & 5.75 & 0.657 & 0.062 & 0.17 \\
& $\ell$ & $1.12 \times 10^{20}$ & 1 & 3.30 & 1 & 0.0823 & 0.0033 & 2.60 & 5.00 & 0.800 & 0.14 & 0.31 \\
\hline \hline & tot & $1.12 \times 10^{20}$ & 1 & 1 & 1 & 4.17 & 0.0130 & 2.59 & 3.50 & 0.800 & 0.023 & 0.075 \\
& $n \ell$ & $1.12 \times 10^{20}$ & 1 & 1 & 1 & 3.01 & 0.0130 & 2.59 & 3.50 & 0.799 & 0.015 & 0.047 \\
\hline
\end{tabular}

${ }^{\dagger} \mathrm{erg} \mathrm{cm}^{-3} \mathrm{~s}^{-1} ;{ }^{\ddagger} \mathrm{erg} \mathrm{cm}^{-3} \mathrm{~K}^{-1}$

the approximations are

$$
\begin{aligned}
\left\{\begin{array}{l}
a^{\prime} \\
b^{\prime}
\end{array}\right\}\left(\frac{\rho_{*}}{m_{0} n_{0}}\right)^{k / 3} & \rightarrow a_{1} \xi^{k} x_{\rho}^{k / 3} \\
K \rho_{*}^{\gamma-1} / c^{2} & \rightarrow\left(a_{2} x_{\rho} \xi^{3}\right)^{\gamma-1}, \\
\gamma & \rightarrow \frac{a_{3}}{1+a_{4} \xi \sqrt{x_{g}^{5} / x_{\rho}}}, \\
\frac{2 G m_{*}}{r_{*} c^{2}} & \rightarrow a_{5} x_{g},
\end{aligned}
$$

where $a_{1}, \ldots, a_{5}$ are the fit parameters to be optimized. They are expected to be different for the different quantities (for $L_{\nu}^{\infty}$ due to different neutrino processes, and for $C_{\text {core }}$ due to different particle fractions).

Note that the expression for $\gamma$ is quite arbitrary to account for the fact that more massive and, consequently, denser stars should contain softer matter. In Eq. (19c), $\gamma$ depends actually on $\xi \sqrt{x_{g}^{5} / x_{\rho}} \propto M^{2} / R_{\text {core }}$, which ensures its reasonable dependence.

It is convenient to introduce

$$
J_{k p}(M, R)=a_{1} \xi^{k-3} \frac{x_{\rho}^{k / 3}\left[1+\left(a_{2} x_{\rho} \xi^{3}\right)^{\gamma-1}\right]^{\frac{p \gamma-k / 3}{\gamma-1}}}{\left(1-x_{g}\right)^{p / 2} \sqrt{1-a_{5} x_{g}}}
$$

with $\xi$ given by Eq. (18) and $\gamma$ by Eq. (19c). Finally, the approximations take the forms

$$
\left\{\begin{array}{c}
L_{\nu}^{\infty}(\widetilde{T}) \\
C_{\text {core }}(\widetilde{T})
\end{array}\right\}=\left\{\begin{array}{c}
Q_{0} \\
c_{0}
\end{array}\right\} R^{3} \widetilde{T}_{9}^{n} J_{k p}(M, R)
$$

The values $n, p$ and $k$ are taken from Eqs. (12) and (13) and listed in Table IV. The dimensionless parameters $a_{1}, \ldots, a_{5}$ in Eq. (20) will be obtained by the calibration to numerical calculations.

\section{Calibration to numerical calculations}

Our numerical results (Sec. IV A) are shown by different symbols in Figs. 46. We have obtained 123 values of $L_{\alpha}^{\infty}$ (for each $\alpha=n n$ and MU) as well as 123 values of $C_{\alpha}$ (for each $\left.\alpha=n, p, \ell, t o t, n l\right)$. For $L_{\mathrm{DU}}^{\infty}$, we have excluded 45 values with $M<1.5 M_{\odot}$. The trial functions $L_{\nu}^{\infty}(M, R)$ and $C_{\text {core }}(M, R)$ (Eqs. (20) and (21)) have been calibrated to these data sets. The target function to be minimized has been the relative root mean square (rms) error. We present also the maximum relative fit errors over thesame data sets. The parameters $Q_{0}, c_{0}$, $n, p$ and $k$ have been taken fixed from the consideration presented above. We have varied $a_{1}, \ldots, a_{5}$ to minimize the rms error. We present the values of the these parameters with minimum number of digits which do not change the rms error taken with two significant digital numbers. The optimized values of $a_{1}, \ldots, a_{5}$ as well as the corresponding fit errors are listed in Table IV] Figures 46 compare the fits with numerical calculations.

Let us discuss the approximations of $L_{\nu}^{\infty}$. They are the most precise for the nn-bremsstrahlung; the rms error appears to be the lowest here because $Q_{n n}$ is independent of the fractions of charged particles in dense matter. The largest errors occur for the MU case due to a strong dependence of $Q_{\mathrm{MU}}$ on the fractions of charged particles through the factor $\Omega$. The approximation of $L_{\mathrm{DU}}^{\infty}$ is more accurate than that of $L_{\mathrm{MU}}^{\infty}$ because $Q_{\mathrm{DU}}$ depends on $n_{e}$ in a relatively simple way.

The importance of the charged particle fractions can be demonstrated by the instructive examples of the BSk20 and HHJ IV EOSs. In Figs. 24 the corresponding curves are plotted by the blue short-dashed (BSk20) and black dot-dashed (HHJ IV) lines. The numerical data in Fig. 4 are displayed by the black squares (BSk20) and black triangles (HHJ IV). According to Fig. 2, these EOSs result in very close maximum masses, but the stars with the BSk20 EOS are more compact, i.e. they have smaller radii than the HHJ IV stars of the same $M$. Roughly 

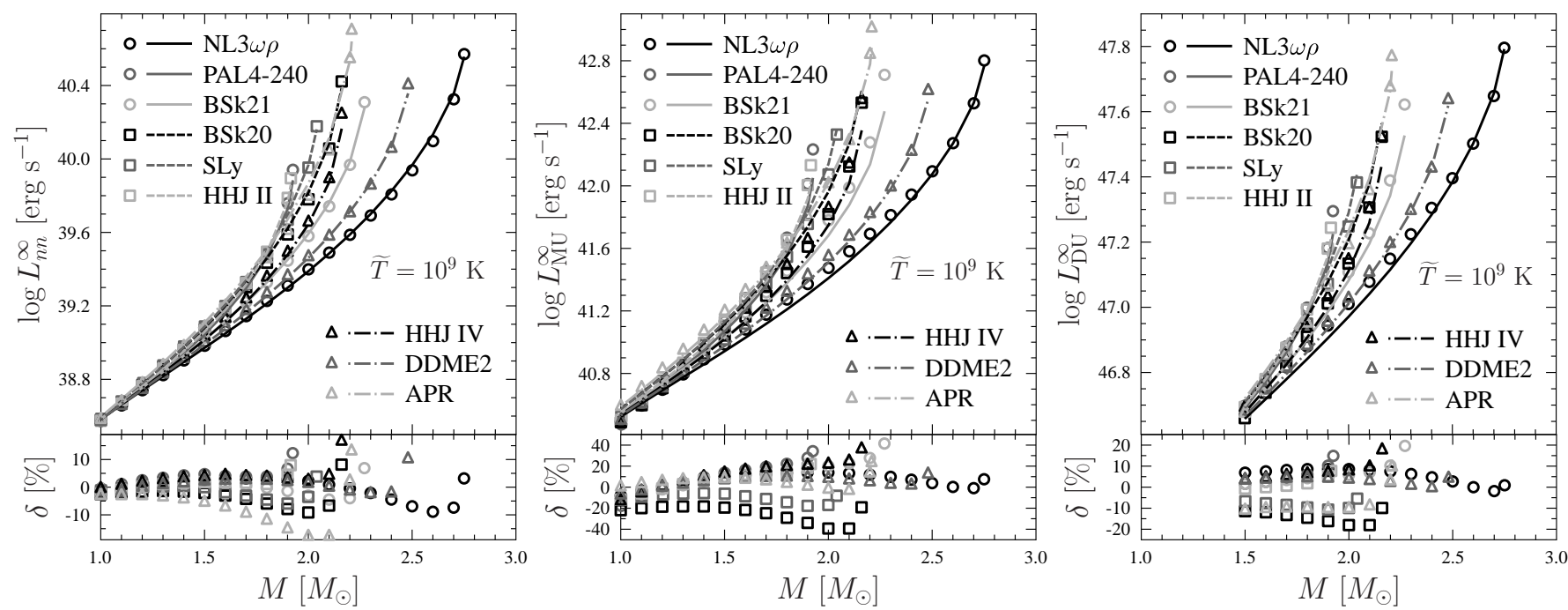

FIG. 4. $L_{\nu}^{\infty}-M$ relations for the nine selected EOSs at $\widetilde{T}=10^{9} \mathrm{~K}$. Lines show the approximation (21); squares, circles and triangles show numerical calculations. For $L_{\mathrm{DU}}^{\infty}$, the DU process is artificially extended over the entire core, but the calculations are performed only at $M \geq 1.5 M_{\odot}$. The bottom panels display the relative fit errors. See text for details.
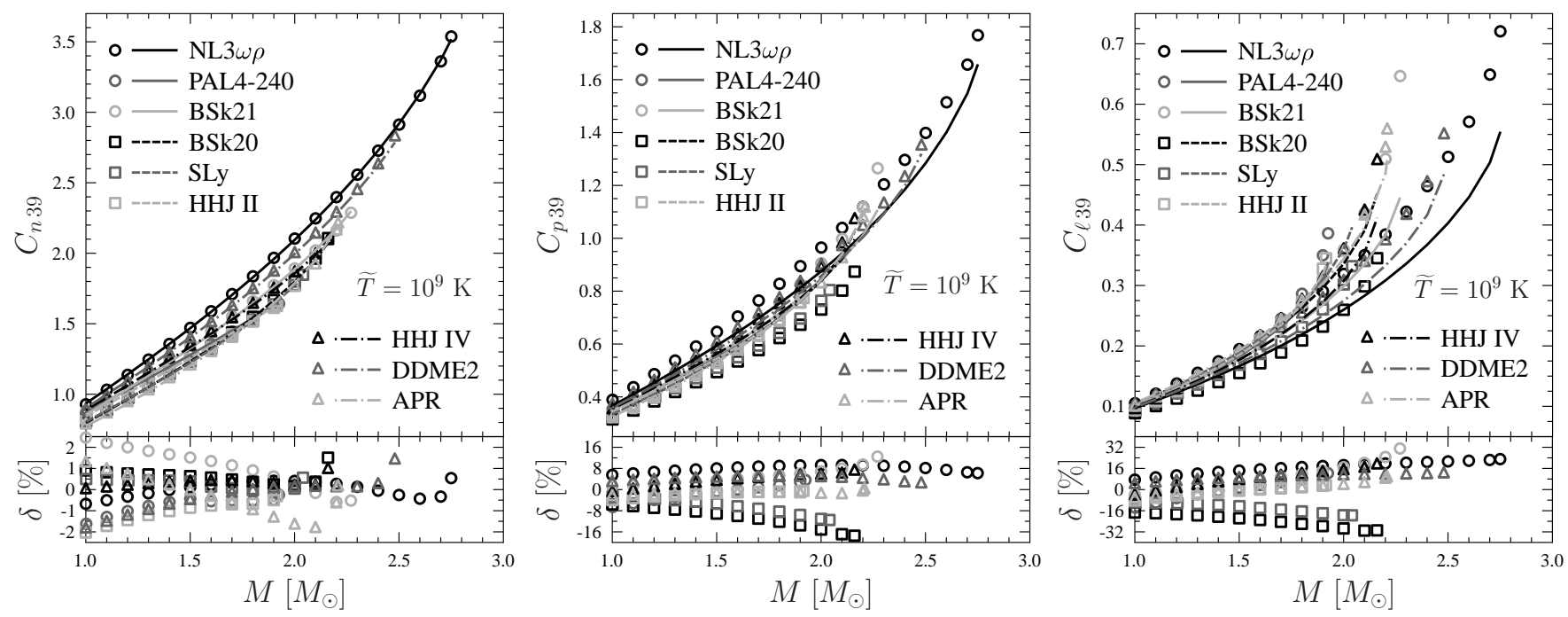

FIG. 5. $C_{\text {core }}-M$ relations for the nine selected EOSs at $\widetilde{T}=10^{9} \mathrm{~K}$, with $C_{\alpha} 39=C_{\alpha} /\left(10^{39} \mathrm{erg} \mathrm{K}^{-1}\right)$ for $\alpha=n, p$ and $\ell$. Lines show the approximation (21); squares, circles and triangles show numerical calculations. The bottom panels display the relative fit errors. See text for details.

speaking, the $M-R$ relations for these EOSs differ by a shift along the $R$ axis. This means that a BSk20 star is denser than an HHJ IV star, and, therefore, has larger $L_{\nu}^{\infty}$. This is really true for $L_{n n}^{\infty}$ (Fig. (4): the squares (for the BSk20 EOS) lie higher than the triangles (for the HHJ IV EOS). This feature is well reproduced by the corresponding black dashed and dot-dashed lines, which show the approximations (21) for these EOSs. In contrast, the MU and DU luminosities are sensitive to the $n_{p}\left(n_{b}\right)$ relations. According to Fig. 3, the values of $n_{p}$ for the HHJ IV EOS are noticeably higher than for the BSk20 EOS. The opposite effects of the two factors, the greater compactness of the BSk20 stars and the larger $n_{p}$ for the HHJ IV stars, lead to their compensation. Accordingly, the DU as well as the MU neutrino luminosities for these EOSs appear to be close enough (the corresponding triangles and squares in the middle and left panels of Fig. (4 overlap). Because the approximation (21) is derived using a not very accurate description of the proton, electron and muon fractions, it cannot reproduce this effect exactly; an approximate expression gives $L_{\mathrm{DU}}^{\infty}$ and $L_{\mathrm{MU}}^{\infty}$ higher than the numerical values for the BSk20 EOS and lower than for the HHJ IV EOS. Moreover, the MU and DU luminosities of the BSk20 and SLy stars are systematically overestimated by the approximation (21) as these EOSs have essentially smaller charged 


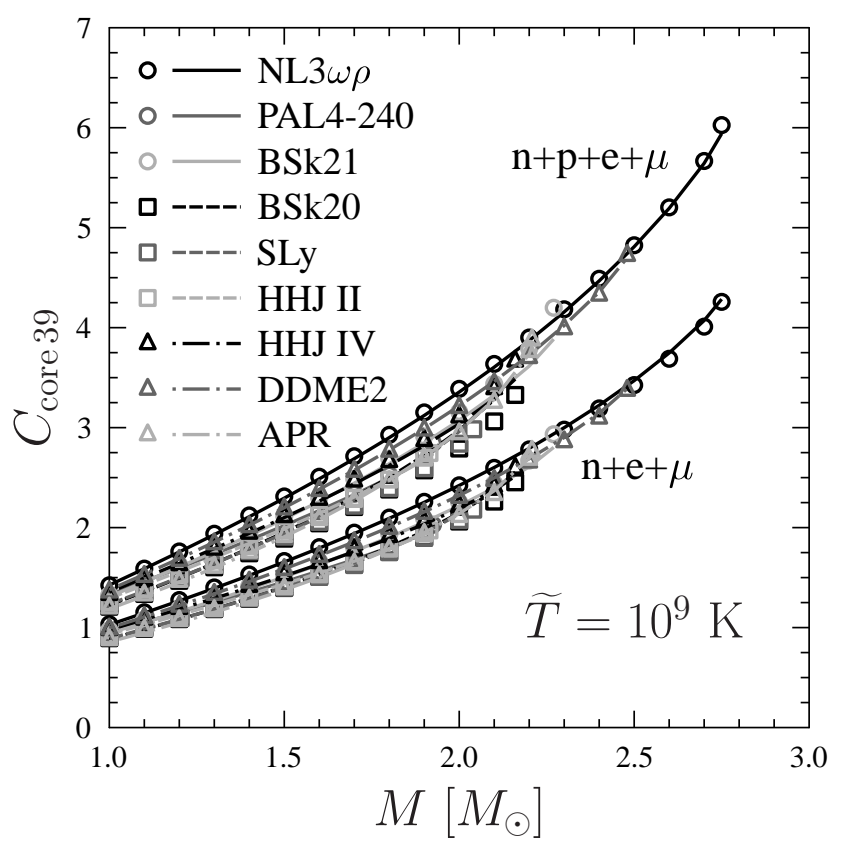

FIG. 6. $C_{\text {core }}$ vs. $M$ for the nine selected EOSs at $\widetilde{T}=10^{9} \mathrm{~K}$; $C_{\text {core } 39}=C_{\text {core }} /\left(10^{39}\right.$ erg $\left.\mathrm{K}^{-1}\right)$. The legend is the same as in Fig. [5] The "n $+\mathrm{p}+\mathrm{e}+\mu$ " = "tot" curves correspond to the fully non-superfluid core. The " $\mathrm{n}+\mathrm{e}+\mu$ " $=$ " $n \ell$ " curve is for the core with strongly superfluid protons but normal neutrons. See text for details.

particle fractions than the other EOSs.

Now let us outline the approximations of the heat capacity (Figs. [5] and 6). The neutron contribution (the left panel of Fig. (5) is accurately reproduced by the approximation (21). It is precise enough to distinguish between very close $C_{n}-M$ relations for different EOSs. On the contrary, the approximations hardly resolve the different curves for $C_{p}$ and $C_{\ell}$. The fit errors are about seven times larger for $C_{p}$ and $12-15$ times larger for $C_{\ell}$ since the details of the $n_{p}\left(n_{b}\right)$ and $n_{e}\left(n_{b}\right)$ relations cannot be well reproduced by the functions which depend on $M$ and $R$ only. Similarly to $L_{\mathrm{MU}}^{\infty}$ and $L_{\mathrm{DU}}^{\infty}$, the numerical values of $C_{p}$ and $C_{\ell}$ for the BSk20 and SLy stars are systematically smaller than the fitted values. Nevertheless, since $C_{n}$ dominates over the other contributions, the approximation (21) almost precisely reproduces $C_{t o t}$ and $C_{n}$ (Fig. 6). The difference between the $a_{1}$ values shows that switching off the proton contribution reduces $C_{\text {core }}$ by about $25 \%$, in agreement with the results of Ref. [25]. Note that the sum of the fits $C_{n}+C_{p}+C_{\ell}$ gives 1-2\% larger errors than $C_{t o t}$, with the parameters from the last two lines in Table IV.

Let us mention several common features of our approximations. First, the index $\gamma$ given by Eq. (19c) with the values of $a_{3}$ and $a_{4}$ from Table IV ranges from 2.3-2.5 for low-mass stars to 1.7-1.9 for high-mass stars. This seems realistic for the considered set of EOSs. Second, the fit errors increase with growing $M$ (except for the almost

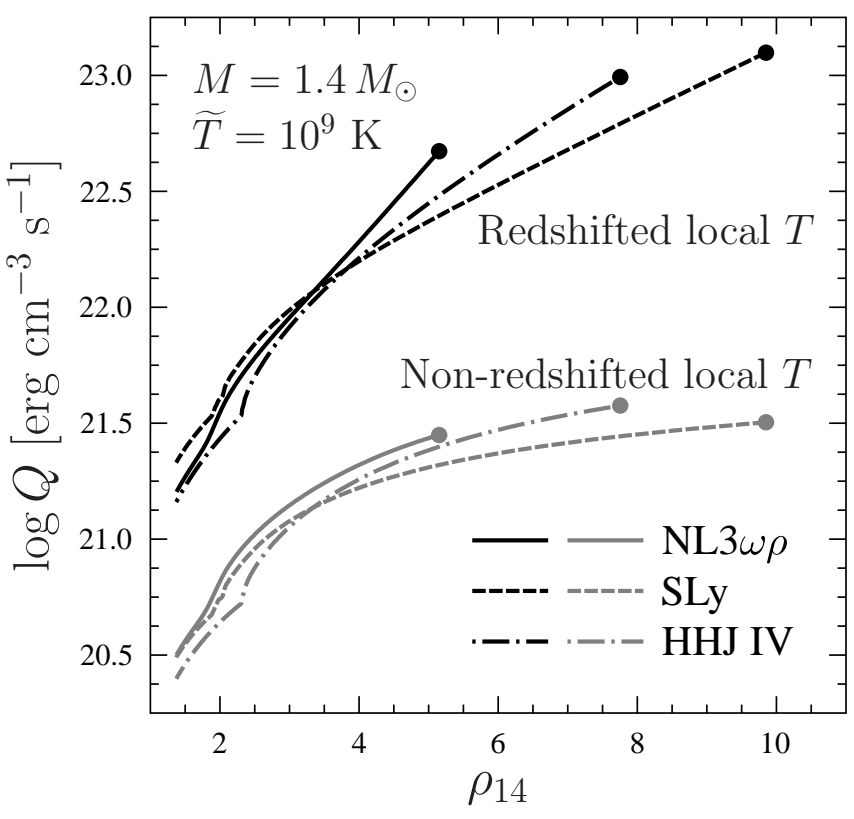

FIG. 7. Logarithm of the neutrino emissivity $Q=Q_{\mathrm{MU}}$ (standard neutrino candle) versus $\rho_{14}=\rho /\left(10^{14} \mathrm{~g} \mathrm{~cm}^{-3}\right)$ in the core of a $1.4 M_{\odot}$ neutron star with three different EOS models. The core is isothermal, with $\widetilde{T}=10^{9} \mathrm{~K}$. The upper (black) lines are calculated using the correctly redshifted local temperature $T$ of the matter. The lower (gray) lines are calculated neglecting the gravitational redshift, that is with $T=\widetilde{T}$. A jump of $Q$ at lower $\rho$ is due to the appearance of muons. Filled circles mark $Q$ and $\rho$ in the center of the star.

precise approximation of the neutron heat capacity), because the higher the density, the stronger the difference between the EOS models.

Our grid of selected EOSs is wide but nevertheless restricted. For instance, $M_{\mathrm{DU}}>1.5 M_{\odot}$ for all of them (Table III), which seems reasonable (e.g., Ref. 41] and references therein) but is not strictly proven. To check "universality" of our fits we have taken the NL3 EOS [26] (with $M_{\mathrm{DU}}=0.84 M_{\odot}$ and $M_{\max }=2.77 M_{\odot}$ ). Some fit errors appear to be somewhat higher while others somewhat lower than for the selected EOSs; nevertheless they seem acceptable. For instance, the maximum relative errors in the $n n, \mathrm{MU}$ and DU cases for stars with the NL3 EOS appear to be 0.07, 0.39 and 0.22 , respectively, while for the selected EOSs we had 0.17, 0.42, and 0.29 (Tables III and IV). For the heat capacities $n, \ell, p$, tot, $n \ell$ we now obtain the maximum errors $0.09,0.22,0.47$, 0.09 , and 0.07 versus $0.025,0.17,0.31,0.075,0.047$ for the selected EOSs. 


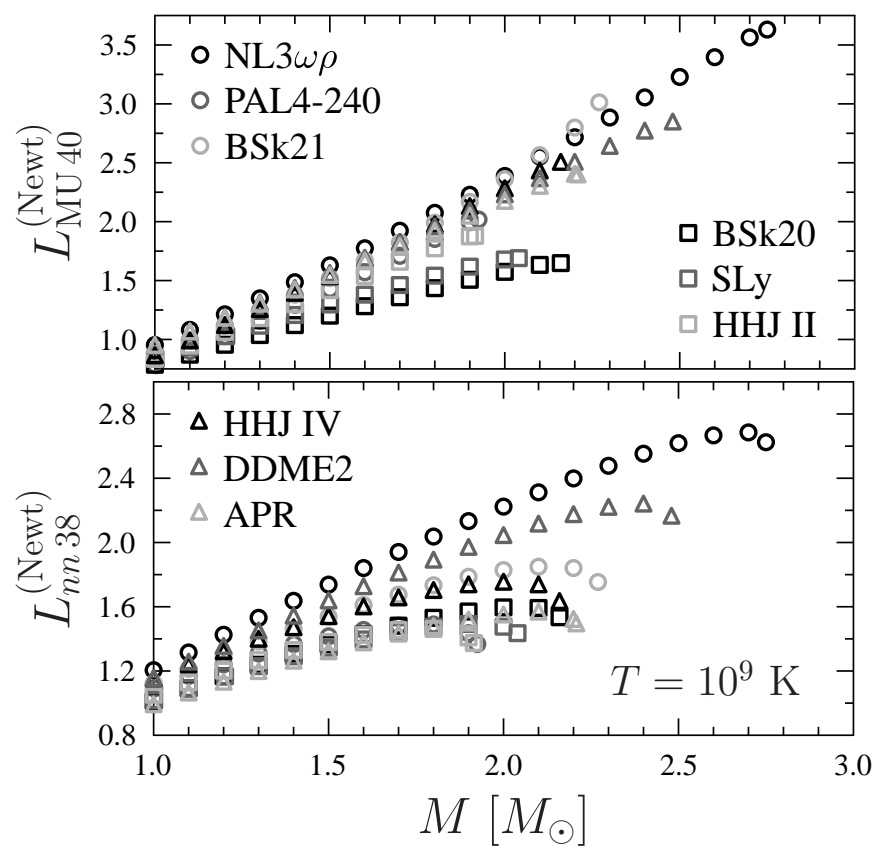

FIG. 8. $L_{\nu}^{\text {(Newt) }}$ vs. $M$ for $n n$-bremsstrahlung (the bottom panel, in $10^{38} \mathrm{erg} \mathrm{s}^{-1}$ ) and MU (the top panel, in $10^{40} \mathrm{erg} \mathrm{s}^{-1}$ ) processes for the nine selected EOS models at $\widetilde{T}=10^{9} \mathrm{~K}$.

\section{IMPORTANCE OF REDSHIFT IN NEUTRON STAR INTERIOR}

While calculating $L_{\nu}^{\infty}$ and $C_{\text {core }}$, it is important to include the proper temperature and emissivity redshifts in a neutron star core.

For instance, Fig. 7 shows the neutrino emissivity $Q=Q_{\mathrm{MU}}(\rho)$ of the standard neutrino candle (due to the MU process) in the nonsuperfluid isothermal core of a neutron star. The star is assumed to have the BSk21 EOS and the mass $M=1.4 M_{\odot}$. The isothermal core temperature is taken to be $10^{9} \mathrm{~K}$ (just for example). The black line is calculated correctly, with $\widetilde{T}=10^{9} \mathrm{~K}$ and the local temperature of the matter $T=\widetilde{T} \exp (-\Phi)$ which is higher, than the isothermal temperature $\widetilde{T}$. The gray line is calculated neglecting the gravitational redshift, using (erroneously and intentionally) a constant local temperature $T=10^{9} \mathrm{~K}$ throughout the core. A kick of the neutrino emissivity at lower $\rho$ is caused by the appearance of muons and the associated muonic MU process (e.g., Ref. [6]). One can see that the General Relativistic redshift is very significant for correctly calculating the neutrino luminosity $L_{\nu}^{\infty}$. It greatly increases the local temperature and the neutrino emissivity in the core. This effect becomes stronger near the center of the star (where the redshift is larger). Therefore, the redshift effect strongly intensifies the integrated neutrino luminosity $L_{\nu}^{\infty}$ and increases the contribution of the central part of the core to $L_{\nu}^{\infty}$.

In order to demonstrate this we have calculated the in- tegrals (11) and (2) ignoring all the factors $\exp \Phi$. Specifically, this means that we use the "Newtonian" thermodynamic equilibrium, $T=$ const. We will call such artificial quantities "Newtonian", $L_{\nu}^{\text {(Newt) }}$ and $C_{\text {core }}^{(\text {Newt) }}$, while the true quantities (1) and (2) will be called "relativistic".

Figure 8 plots the $n n$ and MU "Newtonian" luminosities. Comparing them with the left and middle panels of Fig. 4 we see that $L_{\nu}^{\infty}$ and $L_{\nu}^{\text {(Newt) }}$ differ as functions of $M$. If $M$ varies from $1 M_{\odot}$ to $\sim 3 M_{\odot}$, the "Newtonian" ones change only by a factor of two or three, while the "relativistic" luminosities change by two orders of magnitude. Moreover, $L_{n n}^{\text {(Newt) }}$ becomes nonmonotonic near the maximum masses due to a dramatic decrease of the stellar radius. This phenomenon vanishes for the "relativistic" $n n$ luminosity because the total redshift factor $\exp (-6 \Phi)$ in the integrand of Eq. (1) becomes very large $(\sim 10-100)$ for high-mass stars. Another feature is an inverted ordering of the "Newtonian" $L_{n n}^{\infty}$ with respect to the "relativistic" ones for a fixed value of $M$. Note that this ordering completely breaks for $L_{\mathrm{MU}}^{(\mathrm{Newt})}$ because it depends on the fraction of charged particles. It is rather close to the ordering of the proton number densities at a fixed $n_{b}$ in Fig. 3. Nevertheless, the ordering of the "relativistic" MU luminosities in Fig. 4 does not correlate with the ordering of $n_{p}-n_{b}$ curves in Fig. 3.

Note that the neutron contribution to $C_{\text {core }}^{(\text {Newt })}$ is given by the same integral as $L_{n n}^{(\text {Newt })}$ but with $Q_{0 n n}$ from Eq. (6) replaced by the expression for $c_{0}$ from Eq. (9). Thus the bottom panel of Fig. 8 looks like $C_{n}^{(\text {Newt })}$. Its difference from the 'relativistic' quantity (the left panel of Fig. (5) is not so dramatic as for neutrino luminosities, but

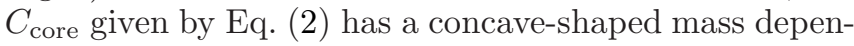
dence, while the curves of the 'Newtonian' heat capacity - mass relation is convex.

Our analysis shows that the true "relativistic" luminosities and heat capacities are dominated by the General Relativistic redshift, as well as by the effects of nuclear and particle physics, like the $n_{p}-n_{b}$ relation and the emissivity dependence on particle number densities.

\section{ILLUSTRATIVE EXAMPLES}

To illustrate our results let us outline a scheme for a possible interpretation of the observations of neutron stars. We have selected nine isolated middle-aged cooling neutron stars listed in Table $\mathrm{V}$. They are PSR J11196127 (hereafter J1119), RX J0822-4300 (in Pup A), PSR J1357-6429 (J1357), PSR B0833-45 (Vela), PSR B170644 (B1706), PSR J0538+2817 (J0538), PSR B2334+61 (B2334), PSR B0531+21 (Crab) and PSR J0205+6449 (in $3 \mathrm{C} 58$ ).

For each of these stars, the ages $t$ and the effective surface temperatures $T_{s}^{\infty}$ have been estimated or constrained. These observational data are presented in Table V together with the references from which the data are taken. Note that the surface temperature of the Crab 
TABLE V. Observational data on nine selected isolated neutron stars which are at the neutrino cooling stage.

\begin{tabular}{lcccccc}
\hline \hline Name & $t[\mathrm{kyr}]$ & $T_{\mathrm{s}}^{\infty}[\mathrm{MK}]$ & $M\left[M_{\odot}\right]$ & $R[\mathrm{~km}]$ & $B[\mathrm{TG}]$ & Ref. \\
\hline PSR J0205+6449 (in 3C 58) & $0.82-5.4$ & $<1.02$ & 1.4 & 12 & 3.6 & {$[\underline{42}, \underline{43}]$} \\
PSR B0531+21 (Crab) & 1.0 & $<2.0$ & 1.4 & 12.14 & 3.8 & $\underline{[20}, \underline{44}]$ \\
PSR J1119-6127 & $0.8-3.2$ & $1.02-1.48$ & 1.4 & 10 & 41 & {$[\underline{45}]$} \\
RX J0822-4300 (in Pup A) & $3.6-5.2$ & $1.6-1.9$ & 1.4 & 10 & 0.033 & {$[\underline{46}-\underline{48}]$} \\
PSR J1357-6429 & $3.65-14.6$ & $0.68-0.86$ & 1.4 & 10 & 7.8 & {$[\underline{49}]$} \\
PSR B0833-45 (Vela) & $11-25$ & $0.65-0.71$ & 1.4 & 10 & 3.4 & {$[\underline{50}]$} \\
PSR B1706-44 & $8.5-34$ & $0.48-0.83$ & 1.4 & 12 & 3.1 & {$[51]$} \\
PSR J0538+2817 & $26-34$ & $0.71-1.07$ & 1.4 & 10.5 & 0.7 & {$[52]$} \\
PSR B2334+61 & $20.5-82$ & $0.55-0.84$ & 1.4 & 10 & 9.9 & {$[45]$} \\
\hline \hline
\end{tabular}

pulsar and the pulsar in 3C 58 is an upper limit only. The surface temperatures have been inferred from the observed spectra of neutron stars using either a blackbody model, or hydrogen atmosphere models (magnetic or nonmagnetic ones). In performing such analyses we have adopted certain values of neutron star mass $M$ and radius $R$ which are also listed in the table. Finally, the magnetic fields of these stars have been mainly estimated from the standard magnetic braking measurements. The values of the surface equatorial magnetic field $B$ were taken from the ATNF pulsar catalogue 153 .

All these neutron stars are thought to be at the neutrino cooling stage with isothermal interiors. Their cooling is regulated by the neutrino cooling function $L_{\nu}^{\infty}(\widetilde{T}) / C_{\text {core }}(\widetilde{T})$ which can be easily calculated from our approximations of $L_{\nu}^{\infty}(\widetilde{T})$ and $C_{\text {core }}(\widetilde{T})$ (Sec. IV]). Here we adopt the minimal cooling paradigm [54, 55], according to which neutron star cores consist of nucleons, muons and electrons (our main assumption throughout this paper) and the DU process is not open there. Following the method developed in our previous studies 14, 15, 20, 22], we can assume certain values of $t, T_{s}^{\infty}, M, R$, and the mass $\Delta M_{\text {acc }}$ of light elements in the heat blanketing envelope and infer the dimensionless cooling function of the neutron star we are studying,

$$
f_{\ell}=\frac{L_{\nu}^{\infty} / C_{\text {core }}}{L_{\mathrm{MU}}^{\infty} / C_{t o t}}
$$

which is the ratio of the actual cooling function to the function for the standard neutrino candle (that is a star of the same mass, radius and EOS but without superfluidity in the core). The magnitude of $f_{\ell}$ is a fundamental parameter of the superdense matter in neutron stars. For a standard candle, $f_{\ell}=1$. Its realistic minimal value is $f_{\ell} \sim 0.01$. It is realized in the presence of strong proton core superfluidity that drastically suppresses the MU

\footnotetext{
${ }^{1}$ www.atnf.csiro.au/people/pulsar/psrcat/
}

process (leaving the $n n$ bremsstrahlung to be the leading neutrino emission mechanism in the core). Its realistic maximum value $f_{\ell} \sim 100$ is realized in the presence of triplet-state neutron pairing which is accompanied by a strong Cooper pairing neutrino emission. Note that the method of extracting $f_{\ell}$ from the observations is not very accurate because of the strong temperature dependence of $L_{\nu}^{\infty} / C_{\text {core }}$ [15].

Therefore, the realistic values of $f_{\ell}$ range from $\sim 0.01$ to $\sim 100$. Since we do not consider the DU process, in many cooling scenarios $f_{\ell}$ is (almost) independent of the time [14] (being just a number that reflects superfluid properties of a cooling neutron star). If $f_{\ell}$ is evaluated for a number of neutron stars, one can generally compare their superfluid properties on the same footing (regardless of their ages) which is a great advantage of this method.

In order to use the method and determine the internal temperature of a star $\widetilde{T}$ from the surface temperature $T_{s}^{\infty}$, we need to specify the mass of light elements (hydrogen, helium and carbon) in the heat blanketing envelope of the star 56]. The matter composed of lighter elements has a larger thermal conductivity (is more heat transparent) which has to be taken into account. Since we would like to account for the effects of the surface magnetic fields $B$ of neutron stars, we employ the $\widetilde{T}-T_{s}$ relation calculated in Ref. [57] for neutron stars with dipole magnetic fields in the heat blanketing envelopes. In such a case, for a given internal temperature, the surface temperature varies along the neutron star surface, and $T_{s}^{\infty}$ means a properly averaged surface temperature that determines the photon thermal luminosity of the star.

The results for nine neutron stars (Table $\mathrm{V}$ ) are plotted in Fig. 9. Each panel corresponds to one of the selected stars and shows allowable values of $f_{\ell}$ vs (unknown) values of $\Delta M_{\mathrm{acc}}$. The horizontal thin hatched lines $\left(\log f_{\ell}=0\right)$ refer to standard candles (to guide the eye). The hatched regions $f_{\ell} \lesssim 0.01$ and $f_{\ell} \gtrsim 100$ have to be excluded because they seem unrealistic from the theoretical point of view (see above). The regions 

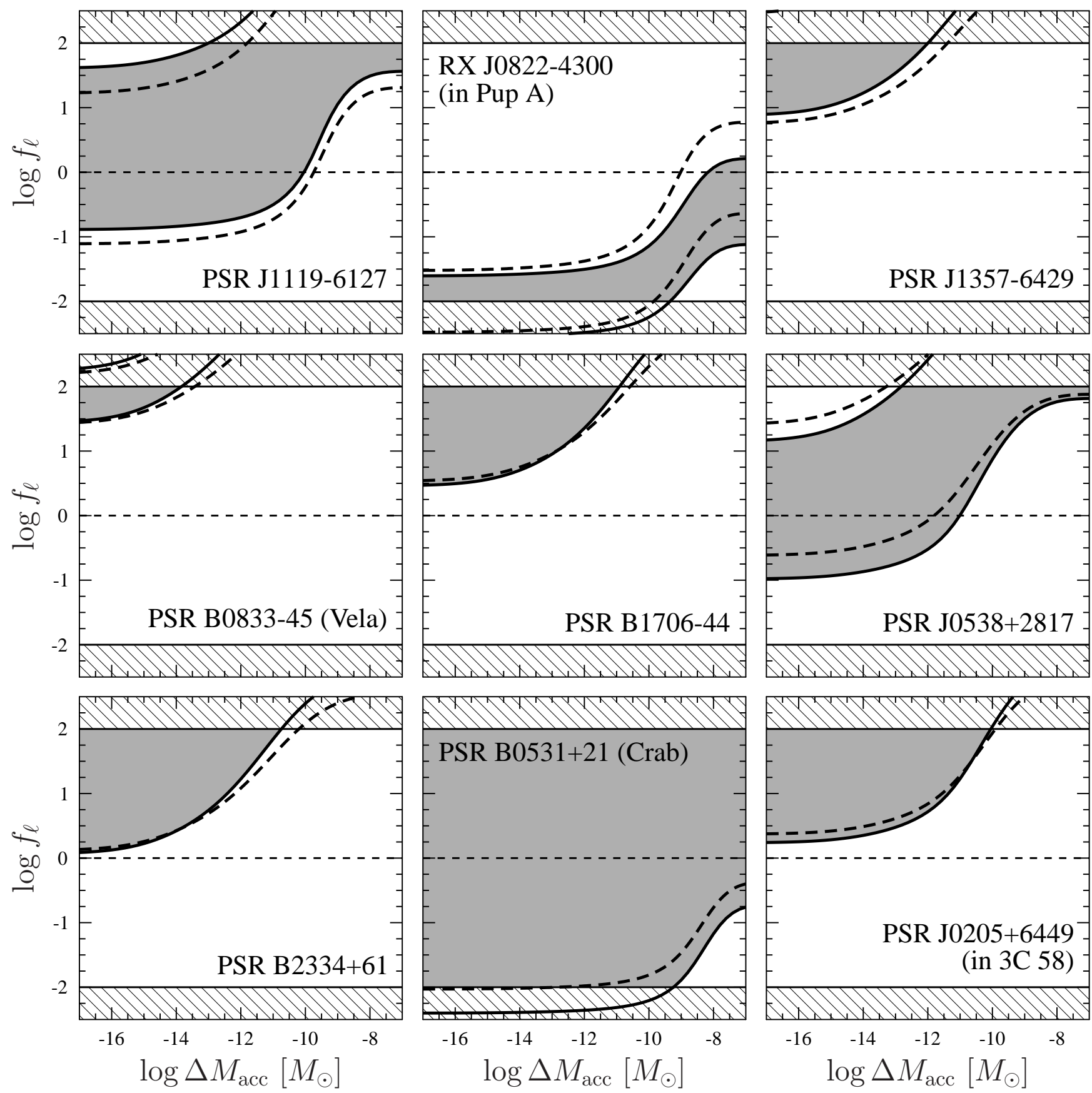

FIG. 9. Neutrino cooling function $f_{\ell}$ vs mass $\Delta M_{\text {acc }}$ of light accreted elements for nine isolated neutron stars at the neutrino cooling stage (Table $\mathrm{V}$ ). The hatched regions exclude too low $\left(f_{\ell}<0.01\right)$ and too high $\left(f_{\ell}>100\right)$ neutrino emission levels which are unreasonable from the theoretical point of view. The short dashed lines refer to the standard neutrino emission level (standard neutrino candle). The grayed regions (restricted mainly by the thick solid lines) show the theoretically accessible ranges of $f_{\ell}$ and $\Delta M_{\text {acc }}$ calculated taking into account the surface magnetic fields. The thick long-dashed lines display the same bound as the thick solid lines but neglecting the effects of $B$ fields. See text for details.

$\Delta M_{\text {acc }} \gtrsim 10^{-7} M_{\odot}$ are also not realistic [56]; the mass of light elements $\Delta M_{\text {acc }}$ cannot be too large (otherwise light elements at the bottom of the heat blanketing envelope will transform into heavier ones under the effect of electron captures and pycnonuclear reactions).
In order to plot Fig. 9, the values of $M, R, T_{s}^{\infty}, t$ and $B$ have been taken from Table $\mathrm{V}$. The grayed regions in the allowable range $0.01 \leq f_{\ell} \leq 100$ are mainly limited by the two thick solid lines calculated including the effects of the magnetic fields. The upper line corresponds to 
the maximum $T_{s}^{\infty}$ and $t$ from Table $\mathbf{V}$, while the lower line is for the minimal $T_{s}^{\infty}$ and $t$. In the cases when the upper line goes much higher than the realistic values of $f_{\ell}$, it is not plotted, and the grayed regions are limited by $f_{\ell}=100$. The thick long-dashed lines are the same as the solid lines but neglecting the effects of the magnetic fields. One can see that the effects are not monotonic and not too strong. These conclusions naturally follow from the results of Ref. [57]. As explained there, the nonmonotonic effects come from the competition of the classical and quantum-mechanical effects of the magnetic field on the thermal conductivity in the heat blanketing envelope of a star.

Let us analyze briefly the results of Fig. 9, First, the mass $\Delta M_{\text {acc }}$ of light elements in the surface layer of a star is very important for inferring $f_{\ell}[20]$. The higher $\Delta M_{\text {acc }}$, the higher $f_{\ell}$ that is required for the same surface temperature and age. Depending on the (generally unknown) $\Delta M_{\text {acc }}$, the same star can be a stronger $\left(f_{\ell}>1\right)$ or weaker $\left(f_{\ell}<1\right)$ neutrino emitter than the standard neutrino candle which would lead to diverging conclusions on its superfluid properties.

Among the nine selected stars, the Crab pulsar is less restrictive to this analysis (Fig. 91). It is young; its thermal surface emission is hidden by a surrounding nebula so that only the upper limit on $T_{s}^{\infty}$ has been established [20]. The solid and long-dashed lines correspond to this upper limit. These results allow the pulsar to have actually any amount of light elements in the surface layers except for a rather large $\Delta M_{\text {acc }} \gtrsim 10^{-10} M_{\odot}$ at a small neutrino cooling function $f_{l} \lesssim 0.1$. These results are in line with those obtained previously in Ref. [20].

For the five other sources (J1357, Vela, B1706, B2334, $3 \mathrm{C} 58)$ we obtain $f_{\ell}>1$, i.e. a stronger neutrino emission than for the case of a standard candle. This situation can occur in the presence of the triplet-state pairing of neutrons in the cores of the five neutron stars. All these stars cannot have a sufficiently large $\Delta M_{\text {acc }}$ (otherwise they would have been too cold). The most restrictive among them is the Vela pulsar. It is so cold that its neutrino cooling function should be close to the maximum theoretical limit, and the amount of light elements in Vela's envelope has to be small. Similar conclusions have been mentioned in Ref. [58].

The other three neutron stars (J1119, Pup A and J0538), depending on $\Delta M_{\mathrm{acc}}$, can have either $f_{\ell}<1$ (which can be explained by rather strong proton superfluidity in their cores) or $f_{\ell}>1$ (which is typically associated with the triplet-state neutron pairing in the bulk of the core).

It is natural to assume that all neutron stars have the same EOS in the core and the same critical temperature profiles, $T_{c n}(\rho)$ and $T_{c p}(\rho)$, for the onset of neutron and proton superfluidities there. Certainly, they can have different masses, radii, $\Delta M_{\text {acc }}$, and magnetic fields. Changing $M$, we vary the widths of the neutron and proton superfluidity layers, and, therefore, $L_{\nu}^{\infty}(\widetilde{T}), C_{\text {core }}(\widetilde{T})$ and $f_{\ell}$. Changing $\Delta M_{\text {acc }}$ and $B$, we modify $\widetilde{T}$ for a given $T_{s}^{\infty}$ which also affects $f_{\ell}$. Therefore, one cannot expect the same $\Delta M_{\text {acc }}$ and $f_{\ell}$ for all neutron stars. By studying the $\Delta M_{\text {acc }}-f_{\ell}$ diagrams, one can investigate statistical distributions of the important parameters of cooling neutron stars. Nevertheless, note that according to Fig. 9, all the selected neutron stars are allowed to have $1 \lesssim f_{l} \lesssim 100$. In the latter case their neutrino emission is stronger than that due to the MU process, and it is consistent with that due to the triplet-state pairing of neutrons in the core. Therefore, no DU process is required to explain the selected cooling neutron stars which is in line with the use of the minimal cooling paradigm. The great advantage of our approach is that it is almost independent of the nucleon EOS in the neutron star core and that it allows us to analyze neutron stars with different $T_{s}^{\infty}$ on the same footing.

If we assumed the operation of the DU processes in the selected neutron stars, our results would be less restrictive. The results would also be different if neutron star cores contain hyperons or exotic matter (like free quarks, or pion or kaon condensates).

\section{CONCLUSIONS}

We have calculated the neutrino luminosities $L_{\nu}^{\infty}(\widetilde{T})$ and the heat capacities $C_{\text {core }}(\widetilde{T})$ in isothermal cores of neutron stars with redshifted internal temperatures $\widetilde{T}$ for nine EOSs (Table III) of superdense matter composed of neutrons, protons, electrons, and muons. We have considered three basic cases of neutrino luminosities (Table (I) and five basic cases of heat capacities (Table III). For any case and any EOS, we have calculated $L_{\nu}^{\infty}(\widetilde{T})$ and $C_{\text {core }}(\widetilde{T})$ for a wide range of masses of neutron stars. The results of these calculations have been approximated by the analytic equations (21) with the parameters given in Table IV, these parameters are independent of the specific EOS. We have shown that $L_{\nu}^{\infty}(\widetilde{T})$ and $C_{\text {core }}(\widetilde{T})$ are strongly affected by the effects of General Relativity (Sec. (V).

Although our analytic approximations are formally independent of EOS in neutron star cores, they are certainly not exact and could be improved in the future. Let us warn that they are obtained for nucleon matter in neutron star cores and cannot be used for the cores with a more complicated composition (containing, for instance, hyperons). Even for the nucleon cores, the approximations are based on a restricted grid of the EOSs and we cannot guarantee that they are sufficiently accurate for many other EOSs. Moreover, our calculations are done using fixed effective masses of nucleons and model expressions for the neutrino emissivities and specific heat capacities. More advanced and reliable calculations of these quantities could change the integrated neutrino luminosities and heat capacities. It is possible that the updated approximations of $L_{\nu}^{\infty}$ and $C_{\text {core }}$ will have the same form (21) but with somewhat different fit param- 
eters. The fit parameters, improved in this way, may depend on specific EOS, which might be taken into account by choosing different parameters (e.g., $a_{1}, \ldots, a_{5}$ ) for different EOSs, but this is the problem for the future. We think, that even now, by a careful analysis of realistic uncertainities introduced by the effects of nuclear physics, one can estimate allowable variations of fit parameters under these effects, but such a complicated problems goes out of the scope of this paper.

For illustration, we have used the approximated $L_{\nu}^{\infty}(\widetilde{T})$ and $C_{\text {core }}(\widetilde{T})$ to analyze the most important neutrino cooling functions $f_{\ell}$, Eq. (22), of nine selected isolated neutron stars (Table V] from observations of their thermal surface emission. We have adopted the minimal cooling paradigm [54, 55] and have determined the allowable ranges of $f_{\ell}$ (Fig. 9) of these stars as functions of the mass $\Delta M_{\text {acc }}$ of light elements in the heat blanketing envelopes of neutron stars and neutron star magnetic fields $B$. While the dependence of $f_{\ell}$ on $\Delta M_{\text {acc }}$ is crucial, the dependence on $B$ turns out to be less important. We have found that the typical values of $f_{\ell}$ for the majority of these stars are higher than 1 (that is higher than for the standard neutrino candle) but lower than 100 (the maximum $f_{\ell}$ that can be provided by the enhanced neutrino emission due to the triplet-state Cooper pairing of neutrons).

Our analysis of observations is definitely incomplete in many respects. We have considered only 9 ordinary, middle-aged, isolated neutron stars whose thermal surface emission and age have been measured (constrained); the total number of detected objects of this type is larger than 20. All the selected stars are at the neutrino cooling stage while some others have already passed to the photon cooling stage. We have taken fixed values of $M$ and $R$ which were mostly assumed to determine $T_{s}^{\infty}$ while fitting the observed spectra with neutron star atmosphere models. It would be more instructive to consider a grid of $M$ and $R$ for each neutron star and use the obtained confidence ranges of $M, R$, and $T_{s}^{\infty}$ (just as in analyzing the data on the neutron star in HESS J1731-347 [15, 22] ). This may result in more accurate values of $T_{s}^{\infty}$ and it may give, additionally, some estimates on $M$ and $R$. Moreover, we could go beyond the minimal cooling paradigm and allow for the appearance of the DU process in massive neutron stars; we could also consider neutron star cores of exotic composition. However, all these problems are beyond the scope of our paper.

The results of the paper can also be used to investigate the thermal states of accreting neutron stars in XRTs (Sec. I).

\section{ACKNOWLEDGMENTS}

DDO and DGY are grateful to Peter Shternin and Valery Suleimanov for useful advices. The work of DGY and DDO was supported by the Russian Science Foundation (grant 14-12-00316) and the work of $\mathrm{PH}, \mathrm{LZ}$ and MF by the Polish NCN research grant no. 2013/11/B/ST9/04528. One of the authors (DDO) is grateful to N. Copernicus Astronomical Center for hospitality and perfect working conditions.
[1] D. G. Yakovlev and P. Haensel, Astron. Astrophys. 407, 259 (2003), astro-ph/0209026.

[2] D. G. Yakovlev, K. P. Levenfish, and P. Haensel, Astron. Astrophys. 407, 265 (2003), astro-ph/0209027.

[3] D. G. Yakovlev and C. J. Pethick, Annu. Rev. Astron. Astrophys. 42, 169 (2004).

[4] D. Page, J. M. Lattimer, M. Prakash, and A. W. Steiner, Astrophys. J. 707, 1131 (2009), 0906.1621.

[5] A. Y. Potekhin, J. A. Pons, and D. Page, Space Sci. Rev. 191, 239 (2015), 1507.06186.

[6] D. G. Yakovlev, A. D. Kaminker, O. Y. Gnedin, and P. Haensel, Phys. Rep. 354, 1 (2001).

[7] E. H. Gudmundsson, C. J. Pethick, and R. I. Epstein, Astrophys. J. 272, 286 (1983).

[8] P. Haensel, A. Y. Potekhin, and D. G. Yakovlev, Neutron Stars. 1. Equation of State and Structure (Springer, New York, 2007)

[9] P. Haensel and J. L. Zdunik, Astron. Astrophys. 227, 431 (1990).

[10] P. Haensel and J. L. Zdunik, Astron. Astrophys. 404, L33 (2003), astro-ph/0305220.

[11] P. Haensel and J. L. Zdunik, Astron. Astrophys. 480, 459 (2008), 0708.3996.

[12] E. F. Brown, L. Bildsten, and R. E. Rutledge, Atrophys.
J. Lett. 504, L95 (1998), astro-ph/9807179.

[13] A. Cumming, E. F. Brown, F. J. Fattoyev, C. J. Horowitz, D. Page, and S. Reddy, Phys. Rev. C 95, 025806 (2017), 1608.07532.

[14] D. G. Yakovlev, W. C. G. Ho, P. S. Shternin, C. O. Heinke, and A. Y. Potekhin, MNRAS 411, 1977 (2011).

[15] D. D. Ofengeim, A. D. Kaminker, D. Klochkov, V. Suleimanov, and D. G. Yakovlev, MNRAS 454, 2668 (2015), 1510.00573.

[16] D. D. Ofengeim, M. Fortin, P. Haensel, D. G. Yakovlev, and J. L. Zdunik, ArXiv e-prints (2016), 1612.04672.

[17] D. Page, J. M. Lattimer, M. Prakash, and A. W. Steiner, in Novel Superfluids, vol. 2,, edited by K. H. Bennemann and J. B. Ketterson (International Series of Monographs on Physics, vol. 157, 505, Oxford University Press, Oxford, 2015), vol. 157, pp. 505-579.

[18] E. Flowers, M. Ruderman, and P. Sutherland, Astrophys. J. 205, 541 (1976).

[19] L. B. Leinson and A. Pérez, Physics Letters B 638, 114 (2006), astro-ph/0606651.

[20] M. C. Weisskopf, A. F. Tennant, D. G. Yakovlev, A. Harding, V. E. Zavlin, S. L. O'Dell, R. F. Elsner, and W. Becker, Astrophys. J. 743, 139 (2011).

[21] P. S. Shternin and D. G. Yakovlev, MNRAS 446, 3621 
(2015), 1411.0150

[22] D. Klochkov, V. Suleimanov, G. Pühlhofer, D. G. Yakovlev, A. Santangelo, and K. Werner, Astron. Astrophys. 573, A53 (2015).

[23] J. M. Lattimer, C. J. Pethick, M. Prakash, and P. Haensel, Physical Review Letters 66, 2701 (1991).

[24] A. D. Kaminker, D. G. Yakovlev, and P. Haensel, Astrophys. Sp. Sci. 361, 267 (2016), 1607.05265.

[25] D. Page, in Nuclear Physics in the Universe, edited by M. W. Guidry and M. R. Strayer (1993), pp. 151-162.

[26] M. Fortin, C. Providência, A. R. Raduta, F. Gulminelli, J. L. Zdunik, P. Haensel, and M. Bejger, Phys. Rev. C 94, 035804 (2016), 1604.01944.

[27] F. Douchin and P. Haensel, Astron. Astrophys. 380, 151 (2001), astro-ph/0111092.

[28] D. Page and J. H. Applegate, Astrophys. J. Lett. 394, L17 (1992).

[29] M. E. Gusakov, A. D. Kaminker, D. G. Yakovlev, and O. Y. Gnedin, MNRAS 363, 555 (2005).

[30] A. Y. Potekhin, A. F. Fantina, N. Chamel, J. M. Pearson, and S. Goriely, Astron. Astrophys. 560, A48 (2013), 1310.0049 .

[31] A. D. Kaminker, A. A. Kaurov, A. Y. Potekhin, and D. G. Yakovlev, MNRAS 442, 3484 (2014).

[32] A. Akmal, V. R. Pandharipande, and D. G. Ravenhall, Phys. Rev. C 58, 1804 (1998).

[33] P. B. Demorest, T. Pennucci, S. M. Ransom, M. S. E. Roberts, and J. W. T. Hessels, Nature 467, 1081 (2010).

[34] J. Antoniadis, P. C. C. Freire, N. Wex, T. M. Tauris, R. S. Lynch, M. H. van Kerkwijk, and et al., Science 340, 448 (2013), 1304.6875.

[35] E. Fonseca, T. T. Pennucci, J. A. Ellis, I. H. Stairs, D. J. Nice, S. M. Ransom, P. B. Demorest, Z. Arzoumanian, K. Crowter, T. Dolch, et al., Astrophys. J. 832, 167 (2016), 1603.00545.

[36] B. L. Friman and O. V. Maxwell, Astrophys. J. 232, 541 (1979).

[37] S. L. Shapiro and S. A. Teukolsky, Black holes, white dwarfs, and neutron stars: The physics of compact objects (Wiley-Interscience, New York, 1983).

[38] O. Y. Gnedin, D. G. Yakovlev, and A. Y. Potekhin, MNRAS 324, 725 (2001).

[39] J. L. Zdunik and P. Haensel, Astron. Astrophys. 530, A137 (2011), 1104.0385.
[40] J. L. Zdunik, M. Fortin, and P. Haensel, Astron. Astrophys. 599, A119 (2017).

[41] M. V. Beznogov and D. G. Yakovlev, MNRAS 452, 540 (2015), 1507.04206.

[42] P. Slane, D. J. Helfand, E. van der Swaluw, and S. S. Murray, Astrophys. J 616, 403 (2004).

[43] Y. A. Shibanov, N. Lundqvist, P. Lundqvist, J. Sollerman, and D. Zyuzin, Astron. Astrophys. 486, 273 (2008).

[44] M. C. Weisskopf, S. L. O'Dell, F. Paerels, R. F. Elsner, W. Becker, A. F. Tennant, and D. A. Swartz, Astrophys. J. 601, 1050 (2004).

[45] V. E. Zavlin, in Astrophysics and Space Science Library, edited by W. Becker (2009), vol. 357 of Astrophysics and Space Science Library, p. 181.

[46] V. E. Zavlin, J. Trümper, and G. G. Pavlov, Astrophys. J. 525, 959 (1999)

[47] W. Becker, T. Prinz, P. F. Winkler, and R. Petre, Astrophys. J. 755, 141 (2012).

[48] E. V. Gotthelf and J. P. Halpern, Astrophys. J. Lett. 695, L35 (2009), 0902.3007.

[49] V. E. Zavlin, Astrophys. J. Lett. 665, L143 (2007).

[50] G. G. Pavlov, V. E. Zavlin, D. Sanwal, V. Burwitz, and G. P. Garmire, Astrophys. J. Lett. 552, L129 (2001).

[51] K. E. McGowan, S. Zane, M. Cropper, J. A. Kennea, F. A. Córdova, C. Ho, T. Sasseen, and W. T. Vestrand, Astrophys. J. 600, 343 (2004).

[52] V. E. Zavlin and G. G. Pavlov, Mem. Soc. Astron. Ital. 75, 458 (2004).

[53] R. N. Manchester, G. B. Hobbs, A. Teoh, and M. Hobbs, Astron. J. 129, 1993 (2005), astro-ph/0412641.

[54] D. Page, J. M. Lattimer, M. Prakash, and A. W. Steiner, Astrophys. J. Suppl. Ser. 155, 623 (2004), astro$\mathrm{ph} / 0403657$.

[55] M. E. Gusakov, A. D. Kaminker, D. G. Yakovlev, and O. Y. Gnedin, Astron. Astrophys. 423, 1063 (2004), astro-ph/0404002.

[56] A. Y. Potekhin, G. Chabrier, and D. G. Yakovlev, Astron. Astrophys. 323, 415 (1997), astro-ph/9706148.

[57] A. Y. Potekhin, D. G. Yakovlev, G. Chabrier, and O. Y. Gnedin, Astrophys. J. 594, 404 (2003), astro$\mathrm{ph} / 0305256$.

[58] M. V. Beznogov, M. Fortin, P. Haensel, D. G. Yakovlev, and J. L. Zdunik, MNRAS 463, 1307 (2016), 1608.08091. 